\title{
Clinical Use of Digital Mammography: The Present and the Prospects
}

\author{
Robert A. Schmidt and Robert M. Nishikawa
}

Digital mammography is likely to replace the current routine breast imaging technology in the future because it offers advantages that should lead to both improved image quality and interpretation. Hopefully. this will result in earlier detection in breast screening programs and decreased mortality from the most frequently diagnosed of all cancers after skin cancer, which is far less deadly. At present, digital mammography has a limited clinical role; in the United States, it has been used for several years to localize lesions for tissue sampling using small field of view digital detectors. Once whole breast digital detectors are available, it seems clear that applying computer techniques to enhance and analyze the collected digital data will become routine. Results reported over the last decade indicate that computer-aided diagnosis can improve radiologists' observational performance, and it is likely that computer techniques to routinely enhance the decision-making ability of the average to belowaverage radiologist to the level of an expert will be developed. There are obstacles to these advances, but the combination of realizable technological solutions and the importance of the breast cancer problem clinically should provide sufficient wherewithal and impetus to make digital mammography a clinical reality.

Copyright $\odot 1995$ by W.B. Saunders Company

KEY WORDS: mammography, breast cancer, digital mammography, computers, diagnostic aid images, digitization.

$\mathbf{T}$ HE CLINICAL ROLE of mammography in detecting and diagnosing early breast cancer has undergone extensive change and expansion over the last 20 years with scientific proof and subsequent public acceptance of routine mammographic screening as the best method known to reduce breast cancer mortality. ${ }^{1} \mathrm{Mam}$ mograms in many radiology departments in the United States have increased by an order of magnitude; volumes at some sites are second

From the Department of Radiology, University of Chicago, Chicago, IL.

Address reprint requests to Robert A. Schmidt, MD, Department of Radiology, MC 2026, University of Chicago, $5841 \mathrm{~S}$ Maryland Ave, Chicago, IL 60637-1470.

This article was adapted and condensed from an article that appeared in Principles \& Practice of Oncology: PPO Updates, ${ }^{6}$ and it is used with the permission of J.B. Lippincott Company.

Copyright $\odot 1995$ by W.B. Saunders Company

0897-1889/95/0801-1021\$3.00/0 only to the chest $x$ ray. State-of-the-art mammography using screen-film methods can reliably detect many small invasive breast cancers, and long-term survival approaches $90 \% .^{2}$ At the University of Chicago, the average size of screendetected nonpalpable invasive breast cancers is now approximately $10 \mathrm{~mm}$ (TNM stage TIb). There has also been a dramatic increase in the diagnosis of preinvasive ductal carcinoma in situ (DCIS), manifested primarily by clustered microcalcifications. Over the past 7 years, $40 \%$ of the nonpalpable cancers found on mammography at my institution have been DCIS, which is essentially curable. Mammography is the most sensitive method of breast cancer detection, finds the smallest breast cancers, and is the only recommended imaging technique for screening of the asymptomatic female population. However, some problems and limitations have emerged over the past decade; not all cancers are detected, primarily because of image quality and observer limitations.

The process of mammographic screening can be subdivided into the primary task of detection and the subsequent task of analysis for clinical significance. Given the small number of breast cancers in a mixed prevalence and incidence population, it is like looking for 5 needles in 1,000 haystacks. This places great demands on the efficiency of detection, and a need to distinguish the myriad normal breast structures from those relatively rare tissue patterns that may signal an underlying malignancy. The $\mathrm{x}$-ray units and technologists are challenged to consistently provide high-quality images to facilitate interpretation, and the radiologists reading these images must be consistently sensitive, while not raising too many false-positive calls. A reasonable goal is to categorize less than $10 \%$ of the screened population as potentially abnormal, and to proceed to tissue diagnosis in less than $2 \%$ after further workup. Whereas human observers have achieved sensitivity rates of about $80 \%$ to $90 \%{ }^{3-5}$ in screening mammography programs, this level of performance is possible only with stringent attention to optimum technical image quality, and it requires a great deal of 
experience on the part of the reader. The success of breast cancer screening calls out for any improvements that might make small tumors more easily visible. As other areas of radiologic practice move to replace film as the primary detector, digital mammography is approaching a stage that may bring it into routine clinical practice within 5 to 10 years. ${ }^{6}$

Mammography has the most demand for image quality of any radiologic procedure. The current trade-off between the need for both high contrast and high resolution is limited by the use of film as both the recording and display medium. Therefore, not all breast cancers can be detected because the image is neither recorded nor displayed optimally. Film has a limited dynamic range in optical densities that can be displayed, and film granularity is an important source of noise that can mask microcalcifications, particularly with extended cycle processing.

\section{PROSPECTS FOR DIGITAL MAMMOGRAPHY}

Digital mammography has the potential to increase the efficacy and accuracy of mammography by reducing errors caused by poor image quality and incorrect human judgment. By uncoupling the detector from the display, increasing the latitude of the detector, and keeping the intrinsic noise level low, the detectibility of cancerous lesions should be improved. A welldesigned digital mammographic system theoretically should have superior image quality to that of a screen-film image for the same radiation dose to the breast. ${ }^{7}$ In part, this is because such a digital system can produce high contrast in all areas of the breast image, whereas conventional images have only a limited area of high contrast. The National Cancer Institute (NCI) sponsored a workshop entitled "Breast Imaging: State-ofthe-Art and Technologies of the Future" in September 1991, and digital mammography was singled out as "the evolving technology with the greatest potential impact on management of breast cancer." 8 One result of the workshop was significant funding by the $\mathrm{NCl}$ beginning in 1993 to establish the National Digital Mammography Development Group (NDMDG). The NDMDG is designed to investigate all the major issues in digital mammography and to facilitate the technology transfer into clinical practice.
The areas of mammographic system design, image-processing, computer-aided diagnosis $(\mathrm{CAD})$, and telemammography will be addressed by the NDMDG members, a consortium of academic and industrial collaborators: the University of Toronto and General Electric Corporation for detector development; the University of North Carolina for image processing; the University of Chicago for computer-aided diagnosis; the General Electric Corporation for telemammography; and Thomas Jefferson University and Massachusetts General Hospital for clinical evaluation. It is expected that there will be clinical testing of the technology within the next 2 to 3 years.

Once the mammographic image has been recorded and transferred to a computer, whether by direct digital acquisition or digitization of mammographic film, it can be processed to improve the image display and enhance the visibility of structures of interest, or analyzed by programs designed to highlight abnormalities. The use of this type of image manipulation is inevitable once the basic transformation of mammographic data into a form digestible by computers becomes routine, and will undoubtedly benefit from the explosion of technology taking place in digital computing. Several investigators have shown that not all of the information in a radiograph is presented in a way that humans can perceive.9-11 Therefore, because current screen-film mammographic images contain information not easily accessible to observers, improving the presentation and display by digitization of film images provides an area for research development that may have significant clinical impact and allows for development of CAD algorithms and testing. This is the current status: image enhancement techniques are being developed using digitized screen-film mammograms, and results are displayed by reprinting the processed image on another film. However, it will not be practical to implement such enhancement methods clinically until a digital workstation for mammography becomes available. ${ }^{12}$

An interesting advantage of digital mammography will be virtually instant image access, rather than the conventional 1.5- to 3-minute delay for standard or extended-cycle rapid automated processing. This has already had an 
impact in interventional procedures, as discussed below, and should significantly improve patient throughput. It is also unlikely that a digital mammogram would have to be repeated as often for improper exposure as is now the case. This is supported by the experience of multiple centers using phosphor-plate technology for chest $\mathrm{x}$ rays taken on intensive-care patients. Another potential benefit is that digital mammograms could be retrieved and reproduced with image quality identical or at least comparable to the original image. This would be a very significant advance when viewed in light of the inadequacy of conventional copy films of mammograms.

\section{CAD}

The area of digital mammography that is generating the most research interest is in CAD, ${ }^{13,14}$ with over 50 research groups world wide. CAD has been defined as a diagnosis made by a radiologist who uses the output of computerized image analyses of the radiograph to help make the decision. ${ }^{15}$ The intent of CAD is to suggest possible lesion locations to the radiologist interpreting the mammogram, and to provide quantitative measures of the malignant potential of lesions. It is not intended to provide an automated diagnosis that would replace the radiologist, but rather can be used as a second opinion by the radiologist, without requiring a second human observer. Several observer studies have advanced the field of CAD in mammography by showing that radiologists' accuracy for detecting lesions can be increased when aided by computerized analysis techniques. Chan et $\mathrm{al}^{16}$ showed that radiologists provided with CAD output that locates clustered microcalcifications improved their detection accuracy significantly over their reading of the mammogram alone. A recent study by Kegelmeyer et a ${ }^{17}$ showed a similar improved result for radiologists in detecting spiculated breast lesions.

Determining whether a mammographic lesion is benign or malignant can be quite difficult. Wu et $\mathrm{a}^{18}$ have shown that radiographic features extracted by radiologists can be used as input to an artificial neural network, with resultant significant improvement over the radiologists initial classification of benign and malig- nant lesions. Other studies ${ }^{19,20}$ have found that general radiologists aided by an expert system could improve their accuracy to the level of expert mammographers. However, these methods require radiologists to extract information from the images and then enter it into the computer. This is impractical for clinical usage, and automated feature extraction techniques must be developed if this approach is to go beyond the laboratory stage.

Promising evidence of the powerful potential of CAD has been shown when programs for mass and microcalcification detection developed at our institution were applied to digitized mammograms in which a significant lesion was present that had been missed by the radiologist reading the examination..$^{21}$ To date, 69 cases ( 38 malignant, 31 benign) in which an observation miss occurred have been analyzed. The CAD schemes were able to identify the lesion in approximately half of the radiologist-missed cases, with 1.3 false-positive detections per image, an acceptable rate. All of the lesions were graded as at least "subtle" (requiring good to expert mammographic experience for detection). This result supports the idea that CAD may have a role as a second reader in mammography, reducing human observation errors. An initial clinical trial of this CAD technique began in 1994 at the University of Chicago, where a workstation (computer, 100- $\mu \mathrm{m}$ laser digitizer, 40-Gbyte magneto-optical jukebox and two 1,024- $\times$ 1,024-pixel monitors) has been installed to digitize all conventional screening mammograms and then to apply the automated detection techniques. A pair of high-resolution monitors will be used as the display mode for indicating the output of the detection algorithms to the interpreting radiologist.

Although studies have shown that CAD can improve radiologists' accuracy, CAD is still at an early stage of development. ${ }^{22}$ All programs to date have been tested on selected cases, and the description of the databases used and relative subtlety of lesions has not always been clear. Large-scale testing of a true clinical crosssection of cases needs to be performed. Furthermore, the observer studies were conducted in simulated clinical environments, and these results need to be reproduced in ordinary clinical 
practice. Research to evaluate this is underway at our own and other centers, but it will take several years of effort to establish clinical utility. The detection programs being developed are also going to improve over the coming years. Experience has shown that even developing algorithms used to detect the skin line (to demarcate areas of the film containing breast tissue from those outside the breast), a task that human observers generally find relatively simple, can involve significant development work. Temporal comparison of screening mammogram studies is also an important technique, as some cancers can only reliably be detected by noting a change from the previous mammogram. It will be clinically necessary to have automated computer techniques do similar comparisons. Correlating lesions in different views of the same breast will also require relatively sophisticated programs to be as good as human observers, particularly for eliminating false detections.

\section{POTENTIAL DISADVANTAGES OF DIGITAL MAMMOGRAPHY}

It is known that high spatial resolution is needed to image fine detail of the borders of microcalcifications and masses in mammograms, which are important diagnostic features. The spatial resolution required for a digital mammogram to be clinically useful is a subject of debate: can breast cancer be diagnosed as accurately from a digital image with $0.1-\mathrm{mm}$ pixel size as it can be from a conventional screen-film mammogram? The ultimate test will involve routine clinical use of the system, determining if there is a significant difference between the two types of images. The argument over the importance of resolution equivalent to current mammograms versus the improved contrast provided by a digital system has not yet been resolved.

Nonetheless, recent clinical digital mammograms have been judged to be of acceptable quality. A study conducted by the FAXIL Group at the University of Leeds compared screen-film mammograms with computed radiography (CR)based digital mammograms of over $4,000 \mathrm{pa}$ tients, using a photostimulable phosphor screen. ${ }^{23}$ This study concluded that "CR is capable of producing mammographic images of sufficiently high quality to allow a radiologist to provide a clinical mammographic service, in which he has at least as much confidence as that which he has with conventional film screen mammography." 24,25 The majority of these mammograms were done as diagnostic studies on patients presenting with a breast problem, and the implications for screening mammography, by far the more common study, are not clear.

A digital mammographic system will be more expensive than a conventional system, with initial estimates of cost in the area of $\$ 250,000$. This additional cost must be weighed against the potential for improved earlier detection resulting in reduced morbidity and mortality, and it must be clinically proven that this potential can be realized and is a sizeable improvement. There are also display and storage problems. At $50-\mu \mathrm{m}$ pixel size, a single digital mammographic exam (2 views of each breast) will contain 160 megabytes of image data. For a digital radiology department where mammography represents about $10 \%$ to $15 \%$ of total exam volume, digital mammographic data manipulation and storage would be over half the total departmental load. Lastly, the most important goal of image processing is to produce an image such that all the information recorded by the detector can be displayed simultaneously. However, the display dynamic range of a CRT monitor, like film, is less than that required. Currently, the best monitors can only display roughly $2,000 \times 2,000$ pixels, and there are no commercially available film printers that can produce a $50-\mu \mathrm{m}$ pixel on a $8-\times 10$-in film, at present. These problems are amenable to technological solution if there is a clinical demand. For example, preset display settings for different mammographic features could be incorporated into a workstation.

\section{CURRENT STATUS OF DIGITAL MAMMOGRAPHY SYSTEMS}

There are two types of digital mammography systems have that been used clinically with some success: storage phosphor and charge coupled device (CCD) based. The first system has not yet achieved any widespread routine clinical 
use, primarily because of a perceived limitation in resolution and noise. The second system has been used clinically since 1992 for performing stereotactic aspiration cytology and core-needle biopsies of nonpalpable breast lesions. ${ }^{26}$ This system uses a conventional mammography phosphor screen to convert the image to light that is recorded not by film, but by a CCD camera, with coupling either via a tapered fiber-optic bundle (Fischer Imaging Company, Denver, CO) or mirrors and lenses (LoRad Corp, Danbury, $\mathrm{CT})$. These systems have adequate spatial resolution (50- $\mu \mathrm{m}$ pixel size), but have limited field of view, a $5-\mathrm{cm}$ square area. In fact, they have been an ideal way to introduce digital techniques into clinical mammographic usage, because the objective is only to target the lesion, not to characterize it. Work is currently underway at several industrial and research laboratories to construct a full-field digital mammography system using similar technology, and early whole-breast images have been displayed at the recent annual meetings of the Radiological Society of North America. Advantages apparent with digital mammographic interventional units are decreased procedure times, and improved conspicuity of calcifications. Using a version of this system, Dershaw ${ }^{27}$ showed that needle localization procedures for breast biopsy could be performed in half the time.

There are two other basic types of system under development, namely area detectors (either amorphous selenium or amorphous silicon) and scanned-beam detectors. ${ }^{28}$ Area detectors have the advantage that they would be used in a fashion similar to that used currently, and would require little if any machine modification. The CR system mentioned above is an example of a digital area detector. Scanning technique is being developed for versions of the commercially available digital stereotactic units to cover the whole breast. Unfortunately, this approach has potential limitations because of a long scan time that may lead to problems with patient motion (which will reduce resolution) and discomfort. Basic research, clinical evaluation and then clinical trials all still need to be performed to determine which, if any, of these approaches may lead to a clinically improved machine.

\section{FUTURE DEVELOPMENTS IN DIGITAL MAMMOGRAPHY}

There are important obstacles to the routine clinical use of digital mammography. Digital mammography promises to produce better clinical images that should improve diagnosis. Although it has had early success in improving stereotactic biopsy procedures, the leap to diagnosis made from whole-breast images is a quantum leap for both the technology and its application. It is very likely that technological advances and clinical research will be able to overcome these obstacles, and provide many or all of the potential benefits described above. Competition between manufacturers makes it likely that a commercial digital mammography system will be available sometime in 1995 or early 1996. However, the inherent advantages of having digital information will not be fully realized until computer algorithms for alignment, processing, detection, extraction, display, and even characterization reach a level of clinical utility and sophistication that are able to help general radiologists currently interpreting mammograms, and, ideally, to raise their abilities to those of an expert. The question is not whether CAD can perform better than human vision or replace radiologists entirely, but whether computer vision can make human vision better. CAD will certainly detect at least some of the lesions missed by human observers, but it remains to be proven that the observers will react appropriately even when the lesion is highlighted, particularly if false-positive rates are high. Screening mammogram volume alone dictates that an improved method of abnormality detection is desirable. Reading screening mammograms is a repetitive task that requires high attention to minute detail, and tasks like that are well suited to automation once digital information is acquired. CAD techniques should provide a second mammographic interpreter that does not suffer from fatigue, irritation, distraction, or boredom in a repetitive task as a human observer does. In a forseeable future, a reading of the screening mammogram might be provided to the patient before she is ready to leave, and mammograms may be bulk processed at distant facilities that have the necessary 
technology and expertise to improve on the current fragmented clinical screening methods, and thereby maximize the known benefit of screening for breast cancer.

\section{REFERENCES}

1. Feig SA: Decreased breast cancer mortality through mammographic screening: Results of clinical trials. Radiology 167:659-665, 1988

2. Tabar L, Fagerberg G, Duffy SW, et al: Update of the Swedish two-county program of mammographic screening for breast cancer. Radiol Clin N Am 30:187-210, 1992

3. Sickles EA: Quality assurance: How to audit your own mammography practice. Radiol Clin N Am 30:265-276, 1992

4. Schmidt RA, Metz CE: Sensitivity of mammography. AJR 154:419-420, 1990 (letter)

5. Bird RE, Wallace TW, Yankaskas BC: Analysis of cancers missed at screening mammography. Radiology 184 : 613-617, 1992

6. Schmidt RA, Nishikawa RM: Digital screening mam mography, in: DeVita VT, Hellman S, Rosenbert SA: Principles and Practice of Oncology PPO Updates, vol 8. Philadelphia, PA, Lippincott, 1994, pp 1-16

7. Holdsworth D, Nishikawa RM, Mawdsley GE, Fenster A, Yaffe MJ: Slot beam digital mammography using a time-delay-integration (TDI) CCD. Proc SPIE 1090:306313,1989

8. Shtern F: Digital mammography and related technologies: A perspective from the National Cancer Institute. Radiology 183:629-630, 1992

9. McSweeny MB, Sprawls P, Egan RL: Enhanced image mammography. AJR 140:9-14, 1983

10. Smathers RL, Bush E, Drace J, et al: Mammographic microcalcification detection with xerography, screen-film and digitized film display. Radiology 159:673-677, 1986

11. Kimme-Smith C: Digital mammography: A comparison of two digitization methods. Invest Radiol 24:869-875, 1989

12. Giles A, Cowen AR, Parkin GHS, et al: A clinical workstation for digital mammography. Proc SPIE 1905:806817,1993

13. Giger ML: Computer-aided diagnosis, in Haus AG, Yaffe MJ (eds): Syllabus: A Categorical Course in Physics. Technical Aspects of Breast Imaging (ed 3). Oak Brook, IL, RSNA Publications, 1994, pp 287-302

14. Vyborny CJ, Giger ML: Review. Computer vision and artificial intelligence in mammography. AJR 162:699-708, 1994

15. Doi K, Giger ML, MacMahon H, et al: Computeraided diagnosis (CAD): Development of automated schemes for quantitative analysis of radiographic images. Semin Ultrasound CT MR 13:140-152, 1992

16. Chan H-P, Doi K, Vyborny CJ, et al: Improvement in radiologists' detection of clustered microcalcifications on mammograms: The potential of computer-aided diagnosis. Invest Radiol 25:1102-1110, 1990

17. Kegelmeyer WP, Pruneda JM, Bourland PD, et al: Computer-aided mammographic screening for spiculated lesions. Radiology 191:131-337, 1994

18. Wu Y, Giger ML, Doi K, et al: Artificial neural networks in mammography: Application to decision making in the diagnosis of breast cancer. Radiology 187:81-87, 1993

19. Cook HM, Fox MD: Application of expert systems to mammographic image analysis. Am J Physiol Imaging 4:1622,1989

20. Getty DJ, Pickett RM, D'Orsi CJ, et al: Enhanced interpretation of diagnostic images. Invest Radiol 23:240 252,1988

21. Schreibman KL, Schmidt RA, Nishikawa RM, et al: Computer-aided diagnosis at mammography: Analysis of 100 lesions missed by radiologists. Radiology 193(P):157, 1994 (abstr, suppl)

22. Vyborny CJ: Can computers help radiologists read mammograms? Radiology 191:315-317, 1994

23. Sonoda M, Takano M, Miyahara J, et al: Computed radiography utilizing scanner laser stimulated luminescence. Radiology 148:833-838, 1983

24. Brettle DS, Ward SC, Parkin GJS, et al: A clinical comparison between conventional and digital mammography utilizing computed radiography. Br J Radiol 67:464, 1994

25. Parkin G: Radiologist's report, in Price J, Brettle DS (eds): UK Department of Health MDD Evaluation Report No. 94/01. London, UK, HMSO, 1994, pp 3-14

26. Schmidt RA: Stereotactic breast biopsy. CA-A Cancer Journal for Clinicians (American Cancer Society) 44:172191, 1994

27. Dershaw DD, Fleischman RC, Liberman L, et al: Use of digital mammography in needle localization procedures. AJR 161:559-562, 1993

28. Yaffe MJ: Digital mammography, in Haus AG, Yaffe MJ (eds): Syllabus: A Categorical Course in Physics. Technical Aspects of Breast Imaging (ed 3). Oak Brook, IL, RSNA Publications, 1994, pp 275-286 